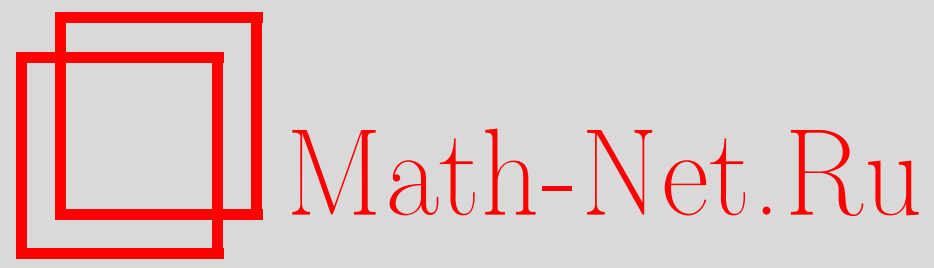

Д. О. Орлов, Квазикогерентные пучки в коммутативной и некоммутативной геометрии, Изв. РАН. Сер. матем., 2003, том 67, выпуск 3, 119-138

DOI: https://doi.org/10.4213/im437

Использование Общероссийского математического портала Math-Net.Ru подразумевает, что вы прочитали и согласны с пользовательским соглашением

http://www.mathnet.ru/rus/agreement

Параметры загрузки:

IP: 3.81 .55 .215

26 апреля 2023 г., 11:46:44 
УДК 512.667

$$
\text { Д.О. Орлов }
$$

\title{
Квазикогерентные пучки в коммутативной и некоммутативной геометрии
}

\author{
К 80-летию Игоря Ростиславовича Нафаревича
}

\begin{abstract}
Дано определение квазикогерентных модулей для любого предпучка множеств на категориях аффинныт коммутативных и некоммутативных схем, что обобщает обычное определение квазикогерентных пучков на схемах. Исследованы свойства квазикогерентных модулей быть пучками в различных топологиях. Построено вложение коммутативной геометрии в некоммутативную с использованием предпучков группоидов.

Библиограбфия: 7 наименований.
\end{abstract}

\section{Введение}

В настоящей статье мы демонстрируем, как квазикогерентные пучки естественньм образом появляются в коммутативной и некоммутативной геометрии. Рассматривая предпучки множеств на категориях аффинных коммутативных и некоммутативных схем как очень широкое обобщение понятия схемы (или алгебраического пространства), показываем, что на этих объектах можно определить квазикогерентные пучки (модули). Далее исследуем основные свойства квазикогерентных пучков на предпучках множеств и, более широко, на предпучках группоидов.

В коммутативной алгебраической геометрии понятие схемы вводится в два этапа. Сначала определяются аффинные схемы, затем произвольная схема склеивается из аффинных кусков. При таком подходе мы апеллируем к топологическим свойствам коммутативных схем. В частности, используется функтор Spec из категории коммутативных алгебр в категорию топологических пространств. С другой стороны, всякая схема может быть рассмотрена как предпучок множеств на категории аффинных схем. Будем использовать именно этот простой факт для введения и исследования некоммутативной геометрии.

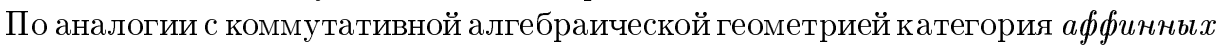
некоммутативных схем - это категория, противоположная категории алгебр (над некоторьм базисным кольцом $k$ ). Всякий объект, который является некоммутативной схемой, должен задавать предпучок множеств на категории аффинных некоммутативных схем.

В настоящей статье рассмотрены все предпучки множеств на категории аффинных схем как в коммутативном, так и в некоммутативном случае. Каждому

Работа выполнена при частичной финансовой поддержке Российского фонда фундаментальных исследований (грант № 02-01-00468), Фонда поддержки ведущих научных школ (грант № 00-15-96085) и INTAS-OPEN (грант № 2000-269). Исследования, описанные в настоящей работе, были сделаны при частичной поддержке Американского фонда гражданских исследований (CRDF RM1-2405-MO-02). 
предпучку множеств $X$ сопоставим категорию (правых) квазикогерентных модулей $\mathrm{Qcoh}_{r}(X)$ на нем (определение 2.2). Определение этой категории не зависит от топологии. Далее, мы покажем, что любой квазикогерентный модуль является пучком в канонической топологии на $X$ (следствие 3.2 и теорема 4.3). В коммутативной ситуации мы определим новую топологию на категории аффинных схем, которая имеет непосредственное отношение к квазикогерентньм модулям (п. 3.7). Функтор ассоциирования пучка в этой топологии (которая называется топологией эффективного спуска) задает эквивалентность на категориях квазикогерентных модулей (теорема 3.6).

Кроме того, мы опишем “правильное" вложение коммутативной геометрии в некоммутативную. Для этого нам придется расширить класс объектов и рассмотреть не только предпучки множеств, но и предпучки группоидов на категории аффинных некоммутативных схем (п. 4.4). Данное вложение удовлетворяет тому свойству, что категория квазикогерентных модулей на объекте $X$ не будет зависеть от того, в какой геометрии мы этот объект рассматриваем: в коммутативной или некоммутативной (теорема 4.2).

Автор благодарит А. Бондала и А. Розенберга за полезные обсуждения, а также А. Суслина, который ответил на многие вопросы.

Большая часть настоящей работы написана во время пребывания автора в Математическом институте Макса Планка (Бонн). Автору приятно выразить свою благодарность Фонду содействия отечественной науки.

\section{§ 1. Предварительные сведения о категориях. Сайты}

1.1. В этом параграфе приведены уже известные факты о категориях и сайтах, которые далее будут использоваться. Все они могут быть найдены в [1] (см. также $[5])$.

Пусть $\mathscr{C}$ - некоторая категория. Обозначим через $\mathscr{C}^{-}$категорию контравариантных функторов из $\mathscr{C}$ в категорию множеств. Объект категории $\mathscr{C}-$ назьвается предпучком множеств на $\mathscr{C}$. Сушествует канонический функтор $h: \mathscr{C} \rightarrow \mathscr{C}^{\widehat{\gamma}}$, который переводит объект $R \in \mathscr{C}$ в функтор $h_{R}(-):=\operatorname{Hom}(-, R)$. Предпучок $h_{R}$ называется представимым. Далее будем писать $R$ вместо $h_{R}$. По лемме Ионеды функтор $h$ является полным вложением, более того, для всякого предпучка $X \in \mathscr{C}^{-}$ сушествует естественный изоморфизм $\operatorname{Hom}_{\mathscr{C}}-(R, X)=X(R)$.

Для произвольного объекта $X \in \mathscr{C}^{-}$обозначим через $\mathscr{C} / X$ категорию пар $(R, \phi)$, где $R \in \mathscr{C}$ и $\phi \in X(R)$. Морфизмы между парами $(R, \phi)$ и $(S, \psi)$ - это морфизмы $f: S \rightarrow R$, для которых $X(f)(\phi)=\psi$. Существует канонический функтор $j_{X}: \mathscr{C} / X \rightarrow \mathscr{C}$, который сопоставляет паре $(R, \phi)$ объект $R$. Отметим, что каждый предпучок $X \in \mathscr{C}^{-}$является копределом представимых объектов по системе $\mathscr{C} / X$ в категории $\mathscr{C}^{-}$.

1.2. Пусть $u: \mathscr{C} \rightarrow \mathscr{C}^{\prime}-$ некоторый функтор. Обозначим через $\widehat{u}^{*}$ функтор из $\mathscr{C}^{{ }^{\prime}}$ в $\mathscr{C}^{-}$, который переводит предпучок $Y \in \mathscr{C}^{\prime-}$ в композицию $Y u$.

Поскольку в категории множеств существуют все малые пределы и копределы, функтор $\widehat{u}^{*}$ имеет левый и правый сопряженные функторы $\widehat{u}_{!}$и $\widehat{u}_{*}$ соответственно (см. [1, гл. I, п. 5.1]). Функтор $\widehat{u}^{*}$ сохраняет все малые пределы и копределы. Функтор $\widehat{u}_{*}$ сохраняет все малые пределы. Функтор $\widehat{u}_{\text {! }}$ сохраняет все малые копределы, и его ограничение на подкатегорию $\mathscr{C} \subset \mathscr{C}^{-}$совпадает с функтором $u$. Кроме того, 
для всякого предпучка $X \in \mathscr{C}^{\widehat{\imath}}$ имеется изоморфизм

$$
\widehat{u} ! X(S) \cong \underset{R \in \mathscr{C} / X}{\lim _{\longrightarrow}} \operatorname{Hom}(S, u R) .
$$

Можно также показать, что функтор $\widehat{u}$ ! является вполне строгим тогда и только тогда, когда функтор $u$ вполне строгий (см. [1, гл. I, п. 5.6]). (Напомним, что функтор $F: \mathscr{C} \rightarrow \mathscr{C}^{\prime}$ называется вполне строгим, если он индуцирует изоморфизм $\operatorname{Hom}_{\mathscr{C}}(Y, X) \stackrel{\sim}{\rightarrow} \operatorname{Hom}_{\mathscr{C}^{\prime}}(F Y, F X)$ для любой пары $Y, X$ объектов категории $\mathscr{C}$.)

1.3. Решетом в категории $\mathscr{C}$ называется полная подкатегория $\mathscr{D} \subset \mathscr{C}$, которая удовлетворяет следуюшему условию: всякий объект из $\mathscr{C}$, для которого существует морфизм в объект из $\mathscr{D}$, сам содержится в $\mathscr{D}$. Пусть $R$ - некоторый объект категории $\mathscr{C}$; тогда решето в категории $\mathscr{C} / R$ будем называть решетом на $R$.

Легко видеть, что решето на $R$ - это то же самое, что подпредпучок предпучка $R$ в категории $\mathscr{C}^{-}$. Поэтому будем рассматривать решето на $R$ как предпучок. Понятие решета необходимо для определения топологии на категории.

ОПРЕДЕЛЕНИЕ 1.1. Топология Гротендика $\mathscr{T}$ на категории $\mathscr{C}$ определяется заданием для каждого объекта $R \in \mathscr{C}$ множества $J(R)$ решет на $R$, называемых покрывающими решетами. Они должны удовлетворять следующим условиям:

1) для любого объекта $R$ максимальное решето $\mathscr{C} / R$ принадлежит $J(R)$;

2) если $T \in J(R)$ и $f: S \rightarrow R$ - произвольный морфизм из $\mathscr{C}$, то решето $f^{*}(T):=$ $\{U \stackrel{\alpha}{\rightarrow} S \mid f \alpha \in T\}$ принадлежит $J(S)$;

$3)$ если $T \in J(R)$ - покрываюшее решето, а $U$ - некоторое решето на $R$ такое, что $f^{*}(U) \in J(S)$ для каждого морфизма $S \stackrel{f}{\rightarrow} R$ из $T$, то $U \in J(R)$.

ОПРЕДЕЛЕНИЕ 1.2. Категория $\mathscr{C}$ с топологией Гротендика $\mathscr{T}$ называется сайтом и будет обозначаться $\Phi=(\mathscr{C}, \mathscr{T})$.

Пусть $\Phi=(\mathscr{C}, \mathscr{T})$ - некоторьй сайт и $R$ - объект из $\mathscr{C}$. Семейство морфизмов $F=\left(f_{\alpha}: R_{\alpha} \rightarrow R\right), \alpha \in I$, называется покрывающим, если решето $T_{F} \hookrightarrow R$, порожденное семейством $F$, является покрывающим решетом на $R$.

ОПреДЕЛЕНИЕ 1.3. Пусть $\mathscr{C}$ - некоторая категория с расслоенньми произведениями. Предтопология на $\mathscr{C}$ определяется заданием для каждого объекта $R$ из $\mathscr{C}$ множества $\operatorname{Cov}(R)$ семейств морфизмов в $R$, удовлетворяющих следующим условиям:

1) семейство с единственным элементом $R \stackrel{\mathrm{id}_{R}}{\rightarrow} R$ принадлежит $\operatorname{Cov}(R)$;

$2)$ если $\left(R_{\alpha} \rightarrow R\right), \alpha \in I$, принадлежит $\operatorname{Cov}(R)$, а $S \rightarrow R-$ морфизм из $\mathscr{C}$, то семейство $\left(R_{\alpha} \times S \rightarrow S\right), \alpha \in I$, принадлежит $\operatorname{Cov}(S)$;

$3)$ если $\left(R_{\alpha} \rightarrow R\right), \alpha \in I$, из $\operatorname{Cov}(R)$ и $\left(R_{\beta_{\alpha}} \rightarrow R_{\alpha}\right), \beta_{\alpha} \in J_{\alpha}$, из $\operatorname{Cov}\left(R_{\alpha}\right)$ для каждого $\alpha \in I$, то семейство $\left(R_{\gamma} \rightarrow R\right), \gamma \in \coprod_{\alpha \in I} J_{\alpha}$, принадлежит $\operatorname{Cov}(R)$.

Всякая предтопология $P$ на $\mathscr{C}$ порождает топологию $\mathscr{T}$ такую, для которой покрываюшими решетами являются решета, содержащие некоторое $P$-покрываюшее семейство.

1.4. Предпучок $X \in \mathscr{C}^{-}$называется пучком (соответственно, отделимымм nредпучком), если для каждого объекта $R \in \mathscr{C}$ и для любого покрывающего решета $S \in J(R)$ канонический морфизм

$$
\operatorname{Hom}_{\mathscr{C}}{ }^{-}(R, X) \rightarrow \operatorname{Hom}_{\mathscr{C}}{ }^{-}(S, X)
$$


является биекцией (соответственно, инъекцией).

Будем обозначать через $\operatorname{Shv}(\mathscr{C}, \mathscr{T})$ категорию всех пучков на сайте $\Phi=(\mathscr{C}, \mathscr{T})$. Категория $\operatorname{Shv}(\mathscr{C}, \mathscr{T})$ является полной подкатегорией в $\mathscr{C}^{-}$.

Функтор вложения $i: \operatorname{Shv}(\mathscr{C}, \mathscr{T}) \rightarrow \mathscr{C}^{-}$имеет левьй сопряженньй функтор $\underline{a}: \mathscr{C}^{\wedge} \rightarrow \operatorname{Shv}(\mathscr{C}, \mathscr{T})$, который известен как функтор ассоциирования пучка, или функтор пучковизации. Функтор $\underline{a}$ сохраняет все конечные пределы и копределы. Для любого предпучка $X$ можно определить предпучок $L X$ по правилу

$$
L X(R)=\underset{T \in J(R)}{\lim _{T \in}} \operatorname{Hom}_{\mathscr{C}}(T, X) .
$$

Это соответствие задает функтор из $\mathscr{C}^{\wedge}$ в себя, которьй обозначим через $L$. Двойная композиция функтора $L$ с собой изоморфна функтору $i \underline{a}$, т.е. существует изоморфизм $\underline{a} X \cong L L X$ (см. $[1$, гл. III] $)$.

1.5. Пусть $\Phi=(\mathscr{C}, \mathscr{T})$ и $\Phi^{\prime}=\left(\mathscr{C}^{\prime}, \mathscr{T}^{\prime}\right)$ - два сайта. $\Phi$ унктор $u: \mathscr{C} \rightarrow \mathscr{C}^{\prime}$ называется непрерьвным, если для любого пучка $Y \in \operatorname{Shv}\left(\mathscr{C}^{\prime}, \mathscr{T}^{\prime}\right)$ предпучок $\widehat{u}^{*}(Y)$ является пучком на сайте $\Phi$. В этом случае $\widehat{u}^{*}$ индуцирует функтор из категории $\operatorname{Shv}\left(\mathscr{C}^{\prime}, \mathscr{T}^{\prime}\right)$ в категорию $\operatorname{Shv}(\mathscr{C}, \mathscr{T})$, который будем обозначать $u^{*}$. Функтор $u^{*}$ имеет левый сопряженный функтор $u !=\underline{a^{\prime}} \widehat{u} !$

Функтор $и: \mathscr{C} \rightarrow \mathscr{C}^{\prime}$ называется конепрерьвным, если для всякого объекта $R \in \mathscr{C}$ и для любого покрываюшего решета $S \hookrightarrow u(R)$ решето на $R$, порожденное всеми морфизмами $Z \rightarrow R$, для которых $u(Z) \rightarrow u(R)$ пропускается через $S$, является покрывающим решетом на $R$. Заметим, что $u$ является конепрерывным, если и только если функтор $\widehat{u}_{*}: \mathscr{C}^{-} \rightarrow \mathscr{C}^{\prime}$ переводит пучки на $\Phi$ в пучки на $\Phi^{\prime}$. Обозначим через $u_{*}$ функтор из $\operatorname{Shv}(\mathscr{C}, \mathscr{T})$ в $\operatorname{Shv}\left(\mathscr{C}^{\prime}, \mathscr{T}^{\prime}\right)$, который является ограничением $\widehat{u}_{*}$ на категорию пучков. Он имеет левый сопряженный $u^{*}=\underline{a} \widehat{u}^{*} i^{\prime}$ (см. $[1$, гл. III]).

Рассмотрим функтор $u: \mathscr{C}^{\prime} \rightarrow \mathscr{C}$ и топологию $\mathscr{T}$ на $\mathscr{C}$. Индуцированной топологией на $\mathscr{C}^{\prime}$ назьвается наибольшая топология, для которой функтор и является непрерывным.

1.6. Зафиксируем некоторый объект $X \in \mathscr{C}^{-}$и рассмотрим категорию $\mathscr{C} / X$ с каноническим функтором $j_{X}: \mathscr{C} / X \rightarrow \mathscr{C}$. Если $\mathscr{T}$ - некоторая топология на $\mathscr{C}$, то она индуцирует некоторую топологию $\mathscr{T}_{X}$ на $\mathscr{C} / X$. Обозначим через $\Phi_{X}$ получившийся сайт $\left(\mathscr{C} / X, \mathscr{T}_{X}\right)$. В [1, гл. III.5] доказано, что решето $S$ на объекте $(Z \rightarrow X)$ является покрывающим в категории $\mathscr{C} / X$ тогда и только тогда, когда решето $\widehat{j_{X !}}(S) \hookrightarrow Z$ является покрывающим в $\mathscr{C}$. Следовательно, функтор $j_{X}: \mathscr{C} / X \rightarrow \mathscr{C}$ непрерывен и конепрерывен одновременно. Получаем три сопряженных функтора:

$$
\begin{aligned}
& j_{X *}: \operatorname{Shv}\left(\mathscr{C} / X, \mathscr{T}_{X}\right) \rightarrow \operatorname{Shv}(\mathscr{C}, \mathscr{T}), \\
& j_{X}^{*}: \operatorname{Shv}(\mathscr{C}, \mathscr{T}) \rightarrow \operatorname{Shv}\left(\mathscr{C} / X, \mathscr{T}_{X}\right), \\
& j_{X !}: \operatorname{Shv}\left(\mathscr{C} / X, \mathscr{T}_{X}\right) \rightarrow \operatorname{Shv}(\mathscr{C}, \mathscr{T}) .
\end{aligned}
$$

Функтор $j_{X *}$ называется функтором прямого образа. Функтор $j_{X}^{*}$ называется функтором обратного образа, или функтором ограничения на $\mathscr{C} / X$. Функтор $j_{X}$ ! называется продолжение нулем на $\mathscr{C}$. 
Пусть $m: Y \rightarrow X-$ морфизм в $\mathscr{C}^{-}$. Он индуцирует функтор $j_{m}: \mathscr{C} / Y \rightarrow \mathscr{C} / X$. По тем же самым причинам, как и выше, сушествуют три функтора

$$
\begin{gathered}
j_{m}^{*}: \operatorname{Shv}\left(\mathscr{C} / X, \mathscr{T}_{X}\right) \rightarrow \operatorname{Shv}\left(\mathscr{C} / Y, \mathscr{T}_{Y}\right), \\
j_{m *}, j_{m !}: \operatorname{Shv}\left(\mathscr{C} / Y, \mathscr{T}_{Y}\right) \rightarrow \operatorname{Shv}\left(\mathscr{C} / X, \mathscr{T}_{X}\right),
\end{gathered}
$$

где $j_{m *}$ и $j_{m !}-$ соответственно правый и левый сопряженные к $j_{m}^{*}$ функторы.

ПреДЛОЖенИЕ 1.4 [1]. Функтор $j_{X !}: \operatorname{Shv}\left(\mathscr{C} / X, \mathscr{T}_{X}\right) \rightarrow \operatorname{Shv}(\mathscr{C}, \mathscr{T})$ может быть представлен как композииия

$$
\operatorname{Shv}\left(\mathscr{C} / X, \mathscr{T}_{X}\right) \stackrel{e_{X}}{\rightarrow} \operatorname{Shv}(\mathscr{C}, \mathscr{T}) / \underline{a} X \rightarrow \operatorname{Shv}(\mathscr{C}, \mathscr{T})
$$

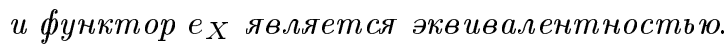

Кроме того, для любого предпучка $X \in \mathscr{C}^{\widehat{\imath}}$ каноническое отображение $m: X \rightarrow \underline{a} X$ задает эквивалентности $j_{m}^{*}$ и $j_{m *}=j_{m !}$ между категориями $\operatorname{Shv}\left(\mathscr{C} / X, \mathscr{T}_{X}\right) u \operatorname{Shv}\left(\mathscr{C} / \underline{a} X, \mathscr{T}_{\underline{a} X}\right)$.

1.7. Топология, для которой каждый представимый предпучок является пучком, называется субканонической; наибольшая такая топология называется канонической. Каноническую топологию будем обозначать сап.

Опишем покрьваюшие семейства для канонической топологии.

Для этого понадобится определение уравнителя. Если $f_{i}: X \rightarrow Y, i=1,2,-$ два морфизма в категории $\mathscr{C}$, то уравнитель - это морфизм $k: K \rightarrow X$, являющийся финальньм в полной подкатегории $\mathscr{C} / X$, состоящей из морфизмов $k^{\prime}: K^{\prime} \rightarrow X$, для которых $f_{1} k^{\prime}=f_{2} k^{\prime}$. Если уравнитель двух морфизмов существует, то он единствен с точностью до изоморфизма.

Предположим, что в категории $\mathscr{C}$ сушествуют все расслоенные произведения. Семейство отображений $\left(X_{\alpha} \rightarrow X\right)_{\alpha \in I}$ в категории $\mathscr{C}$ называется универсальныцм әффективно әпиморфныцм, если для любого отображения $Z \rightarrow X$ и для всякого объекта $Y \in \mathscr{C}$ диаграмма множеств

$$
\operatorname{Hom}(Z, Y) \rightarrow \prod_{\alpha \in I} \operatorname{Hom}\left(Z \times_{X} X_{\alpha}, Y\right) \rightrightarrows \prod_{\alpha, \beta \in I} \operatorname{Hom}\left(Z \times_{X}\left(X_{\alpha} \times_{X} X_{\beta}\right), Y\right)
$$

является уравнителем.

Очевидно, что семейство отображений является покрывающим в канонической топологии тогда и только тогда, когда оно является универсальным эффективно эпиморфным семейством. Пусть $X$ - некоторый объект $\mathscr{C}$. Семейство $\left(W_{\alpha} \rightarrow\right.$ $W)_{\alpha \in I}$ является покрываюшим для объекта $(W \rightarrow X) \in \mathscr{C} / X$ в канонической топологии на $\mathscr{C} / X$ тогда и только тогда, когда оно есть универсальное эффективно эпиморфное семейство в $\mathscr{C}$.

ПРЕДЛОЖЕНИЕ 1.5. Пусть X - предпучок на категории $\mathscr{C}$. Тогда топология на $\mathscr{C} / X$, индуцированная канонической топологией на $\mathscr{C}$ относительно функтора $j_{X}: \mathscr{C} / X \rightarrow \mathscr{C}$, совпадает с канонической топологией на $\mathscr{C} / X$.

Это предложение вытекает из [1, следствие 3.3$]$ и описания покрьвающих семейств в канонической топологии на $\mathscr{C}$ и на $\mathscr{C} / X$. 


\section{§ 2. Окольцованные сайты и квазикогерентные пучки}

2.1. Категория $\mathscr{C}$ с предпучком колец $\mathscr{A}$ называется окольцованной категорией. Сайт $\Phi=(\mathscr{C}, \mathscr{T})$ с пучком колец $\mathscr{A}$ называется окольцованныцм сайтом.

Пусть $\Phi=(\mathscr{C}, \mathscr{T})$ - окольцованный сайт с пучком колец $\mathscr{A}$. Предпучок (правых) $\mathscr{A}$-модулей - это предпучок $\mathscr{M}$ со структурой (правого) модуля над пучком колец $\mathscr{A}$. Если $\mathscr{M}$ является пучком, то будем называть его пучком $($ правы $x) \mathscr{A}-$ модулей.

Обозначим через $\operatorname{Mod}_{r}(\mathscr{A}, \mathscr{T})$ категорию пучков (правых) $\mathscr{A}$-модулей на сайте $\Phi$. Категория $\operatorname{Mod}_{r}(\mathscr{A}, \mathscr{T})$ является абелевой категорией, удовлетворяющей условиям AB5 и $\mathrm{AB} 3^{*}$, и имеет множество порождающих (см. [1, гл. II]).

2.2. Пусть $X$ - объект $\mathscr{C}^{-}$. Категория $\mathscr{C} / X$ с топологией $\mathscr{T}_{X}$, индуцированной функтором $j_{X}: \mathscr{C} / X \rightarrow \mathscr{C}$, образует сайт $\Phi_{X}=\left(\mathscr{C} / X, \mathscr{T}_{X}\right)$. Этот сайт окольцован пучком колец $\mathscr{A}_{X}:=j_{X}^{*} \mathscr{A}$. Будем обозначать категорию пучков (правых)

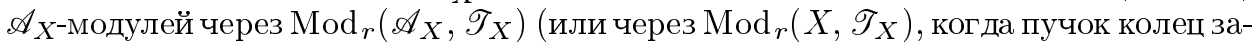
фиксирован).

Пусть $\mathscr{M}$ - пучок (правых) $\mathscr{A}$-модулей. Пучок $j_{X}^{*} \mathscr{M}$ имеет структуру (правого)

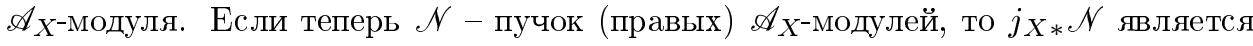

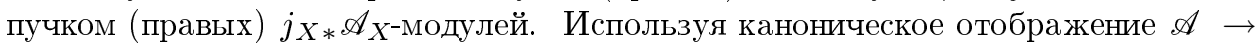
$j_{X * \mathscr{A}}$, получаем, что $j_{X * \mathscr{N}} \mathscr{N}$ имеет структуру (правого) $\mathscr{A}$-модуля. Таким образом, сушествует пара сопряженных функторов

$$
\operatorname{Mod}_{r}\left(\mathscr{A}_{X}, \mathscr{T}_{X}\right) \underset{j_{X *}}{\stackrel{j_{X}^{*}}{\leftrightarrows}} \operatorname{Mod}_{r}(\mathscr{A}, \mathscr{T})
$$

(для функторов между категориями пучков модулей используем те же самые обозначения, как в п. 1.6 для функторов между категориями пучков множеств).

$\Phi$ унктор $j_{X}^{*}: \operatorname{Mod}_{r}(\mathscr{A}, \mathscr{T}) \rightarrow \operatorname{Mod}_{r}\left(\mathscr{A}_{X}, \mathscr{T}_{X}\right)$ имеет также левый сопряженньй функтор $j_{X !}$, который называется продол жение нулем. Отметим, что, в отличие от функторов $j_{X}^{*}$ и $j_{X *}$, композиция функтора $j_{X}$ ! для пучков модулей с забывающим функтором в категорию пучков множеств не совпадает с функтором $j_{X}$ ! для пучков множеств, определенным в п. 1.6 (см. [1, гл. IV, § 11]).

Пусть $m: Y \rightarrow X-$ морфизм в $\mathscr{C}^{-}$. Как и выше, функтор $j_{m}: \mathscr{C} / Y \rightarrow \mathscr{C} / X$ индуцирует три функтора

$$
\begin{aligned}
j_{m}^{*}: \operatorname{Mod}_{r}\left(\mathscr{A}_{X}, \mathscr{T}_{X}\right) & \rightarrow \operatorname{Mod}_{r}\left(\mathscr{A}_{Y}, \mathscr{T}_{Y}\right), \\
j_{m *}, j_{m !}: \operatorname{Mod}_{r}\left(\mathscr{A}_{Y}, \mathscr{T}_{Y}\right) & \rightarrow \operatorname{Mod}_{r}\left(\mathscr{A}_{X}, \mathscr{T}_{X}\right),
\end{aligned}
$$

где $j_{m *}$ и $j_{m}$ ! являются соответственно правым и левым функторами, сопряженными к $j_{m}^{*}$. Более того, функторы $j_{m}^{*}$ и $j_{m *}$ коммутируют с аналогичньми функторами между категориями множеств, определенньми в п. 1.6, относительно забывающих функторов.

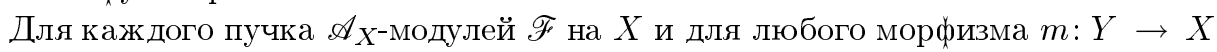
определим пространство сечений пучка $\mathscr{F}$ над $Y$ по формуле

$$
\Gamma(Y, \mathscr{F}):=\operatorname{Hom}\left(\mathscr{A}_{Y}, j_{m}^{*} \mathscr{F}\right) .
$$


ПРЕДЛОЖЕНИЕ 2.1. Пусть $\Phi=(\mathscr{C}, \mathscr{T})-$ сайт, окольцованный пучком колеи А. Для предпучка $X \in \mathscr{C}^{-}$рассмотрим канонический морфизм $m: X \rightarrow$ $\underline{a} X$ ассоциированного пучка. Тогда функторы $j_{m}^{*}$ и $j_{m *}$ межсду категориями $\operatorname{Mod}_{r}\left(\mathscr{A}_{X}, \mathscr{T}_{X}\right)$ и $\operatorname{Mod}_{r}\left(\mathscr{A}_{\underline{a}}, \mathscr{T}_{\underline{a}}\right)$ являются квазиобратимыми әквивалентностями.

Это утверждение следует из предложения 1.4.

2.3. Предположим, что предпучок $X$ представим, т. е. $X \in \mathscr{C}$. Пусть $M-$ (правый) $\mathscr{A}(X)$-модуль. Сопоставим ему предпучок (правых) $\mathscr{A}_{X}$-модулей $\widetilde{M}$ на категории $\mathscr{C} / X$, задаваемый по правилу

$$
\widetilde{M}(S):=M \otimes_{\mathscr{A}(X)} \mathscr{A}(S)
$$

для произвольного объекта $S \rightarrow X$ из $\mathscr{C} / X$.

Теперь рассмотрим произвольный объект $X \in \mathscr{C}^{\widehat{\imath}}$ и соответствующую категорию $\mathscr{C} / X$.

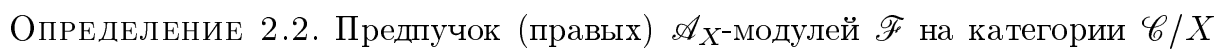
будем называть (правым) квазикогерентным модулем на $X$, если для всякого $m \in X(R)$ предпучок $\hat{j}_{m}^{*}(\mathscr{F})$ на $\mathscr{C} / R$ с канонической структурой (правого) $\mathscr{A}_{R}$-модуля имеет вид $\widetilde{M}$, где $M-($ правый $) \mathscr{A}(R)$-модуль. Квазикогерентный модуль назьвается локально свободным, если для любого $m \in X(R)$ модуль $M$ является проективным.

Морфизмы между квазикогерентньми модулями по определению - это морфизмы между ними как предпучками (правых) $\mathscr{A}_{X}$-модулей. Обозначим $\mathrm{Qcoh}_{r}(X)$ категорию (правых) квазикогерентных модулей на $X$. Она является полной подкатегорией в категории всех предпучков (правых) $\mathscr{A}_{X}$-модулей.

Отметим, что определение квазикогерентного модуля на $X \in \mathscr{C}^{\widehat{ }}$ не зависит от топологии.

2.4. Из определения 2.2 немедленно следует, что для любого представимого объекта $R \in \mathscr{C}$ квазикогерентный пучок изоморфен $\widetilde{M}$ для некоторого $\mathscr{A}(R)$-модуля $M$, т.е. категория $\operatorname{Qcoh}_{r}(R)$ в этом случае эквивалентна категории (правых) $\mathscr{A}(R)$-модулей. Таким образом, предпучок (правых) $\mathscr{A}_{X}$-модулей $\mathscr{F}$ является квазикогерентным на $X \in \mathscr{C}^{\widehat{\gamma}}$ тогда и только тогда, когда для каждого объекта $(R \stackrel{s}{\rightarrow} X) \in \mathscr{C} / X$ предпучок $\hat{j}_{s}^{*} \mathscr{F}$ квазикогерентный на $R$.

2.5. Для всякого $X \in \mathscr{C}^{-}$категория $\mathrm{Q} \operatorname{coh}_{r}(X)$ имеет все коядра и все прямые суммы. Следовательно, она обладает всеми мальми копределами (см. [4, гл. I,, 5$])$.

Пусть $m: Y \rightarrow X-$ морфизм в $\mathscr{C}^{\wedge}$. Функтор $\hat{j}_{m}^{*}:(\mathscr{C} / X)^{\wedge} \rightarrow(\mathscr{C} / Y)^{\wedge}$ индуцирует функтор обратного образа из $\mathrm{Qcoh}_{r}(X)$ в $\mathrm{Qcoh}_{r}(Y)$, который будем обозначать $m^{*}$. Функтор $m^{*}$ точен справа, т. е. он сохраняет коядра. Для квазикогерентного модуля $\mathscr{F}$ на $X$ и для всякого морфизма $m: Y \rightarrow X$ можно определить пространство сечений $\mathscr{F}$ над $Y$ по формуле

$$
\Gamma(Y, \mathscr{F}):=\operatorname{Hom}\left(\mathscr{A}_{Y}, m^{*} \mathscr{F}\right) \text {. }
$$

2.6. Предположим, что топология $\mathscr{T}$ на $\mathscr{C}$ удовлетворяет следующему условию: всякий квазикогерентный модуль на объекте $X \in \mathscr{C}^{-}$является пучком в топологии $\mathscr{T}_{X}$ на $\mathscr{C} / X$. В этом случае категория $\mathrm{Qcoh}(X)$ является полной подкатегорией в $\operatorname{Mod}\left(\mathscr{A}_{X}, \mathscr{T}_{X}\right)$. Обозначим через $\varphi_{X}$ функтор вложения $\operatorname{Qcoh}(X) \hookrightarrow$ $\operatorname{Mod}\left(\mathscr{A}_{X}, \mathscr{T}_{X}\right)$ 
ПРЕДЛОЖЕНИЕ 2.3. Пусть $\Phi=(\mathscr{C}, \mathscr{T})$ - сайт, окольцованный пучком колеи А. Предположим, что все квазикогерентные модули на любом обгекте $X$ являются пучками в топологии $\mathscr{T}_{X}$ на $\mathscr{C} / X$. Пусть $m: X \rightarrow \underline{a} X-$ каноническое отображение ассочиирования пучка для предпучка $X \in \mathscr{C}^{-}$. Тогда функтор обратного образа $m^{*}: \mathrm{Q} \operatorname{coh}(\underline{a} X) \rightarrow \mathrm{Q} \operatorname{coh}(X)$ является вполне строгим.

ДокАЗАТЕЛЬСтво. Сушествует коммутативная диаграмма функторов

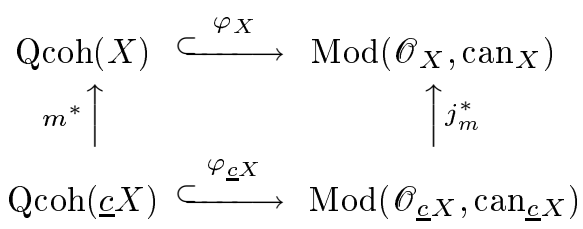

в которой функторы, соответствующие горизонтальньм стрелкам, являются полньми вложениями. Из предложения 2.1 следует, что функтор $j_{m}^{*}$ является эквивалентностью. Следовательно, функтор $m^{*}$ - полное вложение.

2.7. Предположим, что функтор вложения $\varphi_{X}: \operatorname{Qcoh}_{r}(X) \rightarrow \operatorname{Mod}_{r}\left(\mathscr{A}_{X}, \mathscr{T}_{X}\right)$ имеет правый сопряженный функтор $Q_{X}$. Функтор $Q_{X}$ называется когерейтором. Поскольку $\varphi_{X}$ является вполне строгим, композиция $Q_{X} \varphi_{X}$ изоморфна тождественному функтору на $\mathrm{Qcoh}_{r}(X)$. Если $\mathscr{F} \stackrel{f}{\rightarrow} \mathscr{G}$ - некоторьй морфизм в категории $Q \operatorname{coh}_{r}(X)$, то он имеет ядро $\operatorname{Ker}(f)$, которое получается как $Q_{X} \operatorname{Ker}\left(\varphi_{X}(f)\right)$.

Легко проверить, что если когерейтор существует, то категория $\mathrm{Qcoh}_{r}(X)$ является абелевой.

Для представимого объекта $X \in \mathscr{C}$ когерейтор $Q_{X}$ сушествует и является функтором, переводящим пучок $\mathscr{A}_{X}$-модулей $\mathscr{F}$ в квазикогерентный модуль $\widetilde{\mathscr{F}(X)}$.

Пусть $m: Y \rightarrow X-$ морфизм в $\mathscr{C}^{-}$. Если сушествует когерейтор $Q_{X}$, то функтор $m^{*}$ имеет правый сопряженный функтор $m_{*}$, который определяется как композиция $Q_{X} j_{m *} \varphi_{Y}$.

Каждому предпучку $X \in \mathscr{C}^{-}$мы сопоставили категорию (правых) квазикогерентных модулей $\mathrm{Qcoh}_{r}(X)$. Эта конструкция может быть распространена на больший класс объектов в двух направлениях.

2.8. Во-первых, рассмотрим вложение $i: Y \hookrightarrow X$. Определим категорию пары $\mathrm{Qcoh}_{r}(X, Y)$ как полную подкатегорию в $\mathrm{Qcoh}_{r}(X), \operatorname{coc}$ солшую из таких модулей $\mathscr{F}$, для которых $i^{*}(\mathscr{F}) \equiv 0$.

Всякое вложение $i: Y \hookrightarrow X$ индуцирует факторобъект $X / Y$. Это объект с отмеченной точкой, и он определяется как копроизведение

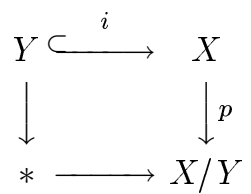

Можно сравнить категории $\mathrm{Qcoh}_{r}(X / Y, *)$ и $\mathrm{Qcoh}_{r}(X, Y)$.

ПРЕДЛОЖЕНИЕ 2.4. Естественный функтор

$$
p^{*}: \mathrm{Q} \operatorname{coh}(X / Y, *) \rightarrow \mathrm{Q} \operatorname{coh}(X, Y)
$$

является әквивалентностью. 
ДокАЗАТЕЛЬСтво. Построим квазиобратньй функтор $p_{*}: \operatorname{Qcoh}_{r}(X, Y) \rightarrow$ $\mathrm{Qcoh}_{r}(X / Y, *)$. Для любого $R \in \mathscr{C}$ имеем равенство $X / Y(R)=X(R) / Y(R)$. Пусть $s \in X / Y(R)$ - сечение. Тогда либо существует единственньй подъем сечения $s$ до сечения $s^{\prime} \in X(R)$, либо оно пропускается через $* \rightarrow X / Y$. Если $\mathscr{F}$ - квазикогерентный модуль на $X$ такой, что $i^{*}(\mathscr{F}) \equiv 0$, то положим $p_{*}(\mathscr{F})(s) \cong \mathscr{F}\left(s^{\prime}\right)$ или $p_{*}(\mathscr{F})(s)=0$, если $s$ - отмеченное сечение. Легко видеть, что таким образом мы получаем функтор, который квазиобратен $p^{*}$.

2.9. Во-вторых, можно рассмотреть не только предпучки множеств, но и предпучки группоидов (или даже предпучки категорий). Под группоидом понимаем малую категорию, в которой все морфизмы обратимы. Малые группоиды образуют 2-категорию. Если отождествим все функторы между группоидами, которые эквивалентны, то получим 1-категорию. Обозначим эту категорию всех малых группоидов через Grd. Всякое множество можно рассматривать как группоид, объекты которого - элементы данного множества, а морфизмами являются только тождественные морфизмы. Каждому группоиду $G$ можно сопоставить два множества: первое, $\mathrm{Ob}(G),-$ множество всех объектов $G$; второе, $\pi_{0}(G),-$ множество связных компонент $G$. Существуют два естественных отображения группоидов ob: $\mathrm{Ob}(G) \rightarrow G$ и $\pi_{0}: G \rightarrow \pi_{0}(G)$.

Обозначим через $\mathscr{C}_{\text {gr }}$ категорию предпучков группоидов на $\mathscr{C}$, т. е. категорию всех контравариантных функторов из $\mathscr{C}$ в категорию малых группоидов Grd. Она содержит категорию $\mathscr{C}^{\wedge}$ в качестве полной подкатегории. Всякий предпучок $X \in$ $\mathscr{\mathscr { C }}_{\mathrm{gr}}$ определяет два предпучка множеств $\mathrm{Ob}(X)$ и $\pi_{0}(X)$, заданных по правилу

$$
\mathrm{Ob}(X)(R)=\mathrm{Ob}(X(R)), \quad \pi_{0}(X)(R)=\pi_{0}(X(R)), \quad R \in \mathscr{C} .
$$

Имеются естественные морфизмы оb $\mathrm{Ob}(X) \rightarrow X$ и $\pi_{0 X}: X \rightarrow \pi_{0}(X)$ между предпучками группоидов. Эти соответствия задают два функтора $\mathrm{Ob}$ и $\pi_{0}$ из $\mathscr{C}_{\mathrm{gr}}^{\widehat{\gamma}}$ в $\mathscr{C}^{-}$.

Морфизм $\pi_{0 X}: X \rightarrow \pi_{0}(X)$ расшепляется для каждого $X \in \widehat{\mathscr{C}_{\mathrm{gr}}}$. (Действительно, выбирая для каждого компонента связности некоторый представитель, получаем расщепление.) Функтор $\pi_{0}: \widehat{\mathscr{C}_{\mathrm{gr}}} \rightarrow \widehat{\mathscr{C}}$ является одновременно правым и левьм сопряженным к каноническому вложению $\mathscr{C}^{-} \rightarrow \mathscr{C}_{\mathrm{gr}}$.

$\mathrm{C}$ каждьм сайтом $\Phi=(\mathscr{C}, \mathscr{T})$ можно связать категорию пучков группоидов $\operatorname{Shv}_{\mathrm{gr}}(\mathscr{C}, \mathscr{T})$ на нем. Предпучок группоидов $X \in \mathscr{C}_{\mathrm{gr}}^{-}$называется пучком, если для любого группоида $G \in \mathrm{Grd}$ предпучок множеств

$$
R \mapsto \operatorname{Hom}_{\mathrm{Grd}}(G, X(R))
$$

является пучком.

2.10. Рассмотрим сайт $\Phi=(\mathscr{C}, \mathscr{T})$, окольцованный пучком $\mathscr{A}$. Для любого предпучка категорий (в частности, предпучка группоидов) определим категорию $\mathscr{C} / X$. Множество объектов $\mathscr{C} / X$ - это множество всех $s \in \mathrm{Ob}(X(S)), S \in \mathscr{C}$. Морфизмы из $s \in \mathrm{Ob}(X(S))$ в $r \in \mathrm{Ob}(X(R))$ - это пары $(\alpha, f)$, где $f: S \rightarrow R$ из категории $\mathscr{C}$ и $\alpha$-некоторьй морфизм из $s$ в $X(f)(r)$ в категории $X(S)$. Существует канонический функтор $j_{X}: \mathscr{C} / X \rightarrow \mathscr{C}$. Индуцируя топологию, получаем сайт $\Phi_{X}=\left(\mathscr{C} / X, \mathscr{T}_{X}\right)$, окольцованный пучком $\mathscr{A}_{X}=j_{X}^{*} \mathscr{A}$. Можно ввести категорию пучков $\mathscr{A}_{X}$-модулей $\operatorname{Mod}_{r}\left(\mathscr{A}_{X}, \mathscr{T}_{X}\right)$. Кроме того, распространяя определение 2.2 , получаем категорию $\operatorname{Qcoh}_{r}(X)$ квазикогерентных модулей на $X$. Mopфизмы $\mathrm{ob}_{X}$ и $\pi_{0 X}$ индуцируют функторы обратного образа оb ${ }_{X}^{*}: \mathrm{Qcoh}(X) \rightarrow \mathrm{Qcoh}(\mathrm{Ob}(X))$ и $\pi_{0 X}^{*}: \mathrm{Q} \operatorname{coh}\left(\pi_{0}(X)\right) \rightarrow \mathrm{Q} \operatorname{coh}(X)$ соответственно. 


\section{§3. Пучки в коммутативной геометрии}

3.1. Зафиксируем коммутативное кольцо $k$. Обозначим через Comalg $/ k$ категорию коммутативных алгебр над $k$ и через Aff $/ k$ - категорию аффинных схем, противоположную Comalg / $k$. Отметим, что категория аффинных схем имеет все расслоенные произведения, соответствуюшие тензорным произведениям в категории Comalg $/ k$. Каноническое вложение категории аффинных схем в категорию всех схем Sch / $k$ coхраняет расслоенные произведения и конечные несвязные объединения, однако оно не сохраняет копроизведения. Например, бесконечное несвязное объединение $\left\{\operatorname{Spec}\left(R_{\alpha}\right)\right\}_{\alpha \in I}$ в $\operatorname{Sch} / k$ не совпадает со $\operatorname{Spec}\left(\prod_{\alpha \in I} R_{\alpha}\right)$.

3.2. В этом параграфе под категорией $\mathscr{C}$ будем понимать категорию всех аффинных схем Aff $/ k$. Канонический контравариантный функтор Aff $/ k \rightarrow$ Comalg $/ k$ задает предпучок колец $\mathscr{O}$ на категории Aff / $k$ по правилу

$$
\mathscr{O}(\operatorname{Spec}(R)):=R
$$

для всякой $k$-алгебры $R$. Этот предпучок представляется групповой схемой Spec $k[t]$. Следовательно, он является пучком в любой субканонической топологии на $\mathscr{C}$.

Таким образом, для любого объекта $X \in \mathscr{C}^{\wedge}$ категория $\mathscr{C} / X$ окольцована предпучком $\mathscr{O}_{X}$, который является пучком для всякой субканонической топологии.

3.3. Обозначим через сап каноническую топологию на $\mathscr{C}=\operatorname{Aff} / k$ и через $\underline{c}: \mathscr{C}^{\widehat{-}} \rightarrow \operatorname{Shv}(\mathscr{C}$, can $)$ - функтор ассоциирования пучка, определенный в п. 1.4, который является левым сопряженным к функтору включения $i: \operatorname{Shv}(\mathscr{C}$, can $) \rightarrow \mathscr{C}^{-}$. Покрывающими семействами для канонической топологии являются универсальные эффективно эпиморфные семейства, определенные в п. 1.7. В случае категории аффинных схем существует простое, но полезное описание для универсальных эффективно эпиморфных семейств в терминах модулей.

ПРЕДЛОЖЕНИЕ 3.1. Семейство $\left(f_{\alpha}: \operatorname{Spec}\left(R_{\alpha}\right) \rightarrow \operatorname{Spec}(R)\right)_{\alpha \in I}$ является универсальным әффективно эпиморфным в $\mathrm{Aff} / k$ тогда и только тогда, когда для каждого $R$-модуля $M$ диаграмма

$$
M \rightarrow \prod_{\alpha \in I} M \otimes_{R} R_{\alpha} \rightrightarrows \prod_{\alpha, \beta \in I} M \otimes_{R}\left(R_{\alpha} \otimes R_{\beta}\right)
$$

является уравнителем.

ДокаЗАТЕЛЬСТво. 1) Возьмем $R$-модуль $M$ и рассмотрим модуль $S=R \oplus M$ как $R$-алгебру с законом умножения, заданным по правилу

$$
\left(r_{1}, m_{1}\right)\left(r_{2}, m_{2}\right)=\left(r_{1} r_{2}, r_{1} m_{2}+m_{1} r_{2}\right) .
$$

Обозначим через $S_{\alpha}$ алгебру $S \otimes_{R} R_{\alpha}$. Рассмотрим семейство $\left(g_{\alpha}: \operatorname{Spec}\left(S_{\alpha}\right) \rightarrow\right.$ $\operatorname{Spec}(S))_{\alpha \in I}$, где $g_{\alpha}=1_{S} \otimes f_{\alpha}$. Оно является эффективно эпиморфньм. Следовательно, вычисляя морфизмы в схему $\operatorname{Spec}(k[t])$, получаем, что

$$
S \rightarrow \prod_{\alpha \in I} S \otimes_{R} R_{\alpha} \rightrightarrows \prod_{\alpha, \beta \in I} S \otimes_{R}\left(R_{\alpha} \otimes R_{\beta}\right)
$$


является уравнителем. Значит, для модуля $M$ как для прямого слагаемого это также выполнено.

2) Пусть $\left(f_{\alpha}: \operatorname{Spec}\left(R_{\alpha}\right) \rightarrow \operatorname{Spec}(R)\right)_{\alpha \in I}-$ некоторое семейство. Известно, что для каждого морфизма $\operatorname{Spec}(S) \rightarrow \operatorname{Spec}(R)$ последовательность (3.2) точна. Значит, для любой алгебры $A$ последовательность гомоморфизмов из $A$ в последовательность (3.2) в категории коммутативных алгебр также точна. Следовательно, $\left(f_{\alpha}\right)_{\alpha \in I}$ является универсальным эффективно эпиморфным семейством.

Как и ранее, для любого предпучка $X \in \mathscr{C}^{-}$будем обозначать через $\mathrm{Qcoh}(X)$ категорию квазикогерентных модулей на $X$ и через $\operatorname{Mod}\left(X, \operatorname{can}_{X}\right)$ - категорию пучков $\mathscr{O}_{X}$-модулей в канонической топологии на $\mathscr{C} / X$.

СлеДСТВИЕ 3.2. Пусть $X$ - некоторый предпучок множеств на категории $\mathscr{C}=\operatorname{Aff} / k$. Всякий квазикогерентный модуль на $X$ является пучком в канонической топологии на категории $\mathscr{C} / X$. Значит, категория $\mathrm{Qcoh}(X)$ является полной подкатегорией в $\operatorname{Mod}\left(X, \operatorname{can}_{X}\right)$.

Это утверждение следует из предложения 3.1 и предложения 1.5.

Обозначим через $\varphi_{X}$ вложение $\mathrm{Q} \operatorname{coh}(X) \hookrightarrow \operatorname{Mod}\left(X, \operatorname{can}_{X}\right)$.

3.4. Категория всех схем Sch $/ k$ вкладывается как полная подкатегория в $\mathscr{C}^{-}=$ (Aff $/ k$ ) очевидньм образом: схеме $X$ соответствует предпучок множеств, значение которого на аффинной схеме $\operatorname{Spec}(R)$ совпадает с $\operatorname{Hom}(\operatorname{Spec}(R), X)$. Для любой схемы $X \in \mathrm{Sch} / k$ квазикогерентные модули в нашем определении однозначно соответствуют квазикогерентным пучкам в обычном определении. Действительно, по каждому квазикогерентному пучку $\mathscr{F}$ на схеме $X$ можно построить квазикогерентный модуль $F$ на $\mathscr{C} / X$ по правилу

$$
F(\operatorname{Spec}(R))=\Gamma\left(\operatorname{Spec}(R), m^{*} \mathscr{F}\right)
$$

для каждого $m: \operatorname{Spec}(R) \rightarrow X$. Это задает эквивалентность, квазиобратную к которой можно определить следующим образом. Для квазикогерентного модуля $F$ на $\mathscr{C} / X$ зададим квазикогерентный пучок $\mathscr{F}$ на схеме $X$ по формуле

$$
\mathscr{F}(U)=\Gamma\left(U, m^{*} F\right)=\operatorname{Hom}\left(\mathscr{O}_{U}, m^{*} F\right)
$$

для каждого вложения $m: U \hookrightarrow X$ открытого по Зарискому множества. Некоторые рассуждения, которые мы опускаем, требуются, чтобы показать, что $\mathscr{F}$ является пучком в топологии Зариского.

Если схема $X$ квазикомпактна и квазиотделима, то сушествует когерейтор $Q_{X}$ : $\operatorname{Mod}\left(X, \operatorname{can}_{X}\right) \rightarrow \operatorname{Qcoh}(X)$. Для категории пучков $\mathscr{O}_{X}$-модулей на малом сайте Зариского это доказано в [2, гл. II.3]. В рассматриваемой ситуации существование когерейтора доказывается аналогичным образом. Действительно, при данных условиях на схему категория $\mathrm{Qcoh}(X)$ имеет множество порождаюших и все копределы. Поскольку вложение $\varphi_{X}$ сохраняет копределы, теорема о сопряженном функторе обеспечивает существование правого сопряженного $Q_{X}$.

ПРЕДЛОЖЕНИЕ 3.3. Пусть $X-$ предпучок на категории $\mathscr{C}=\operatorname{Aff} / k u$

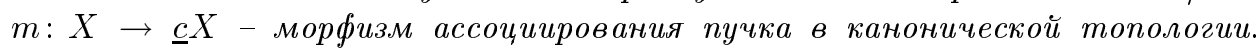
Тогда функтор обратного образа $\mathrm{m}^{*}: \mathrm{Q} \operatorname{coh}(\underline{c} X) \rightarrow \mathrm{Q} \operatorname{coh}(X)$ является вполне строгим.

Это утверждение следует из предложения 2.3 и следствия 3.2 .

5

Серия математическая, №3 
ПРЕДЛОЖЕНИЕ 3.4. Пусть $T \stackrel{i}{\hookrightarrow} \operatorname{Spec}(R)$ - решето на объекте $\operatorname{Spec}(R) \in \mathscr{C}$. Решето Т является покрывающим в канонической топологии тогда и только тогда, когда функтор $i^{*}: \operatorname{Qcoh}(\operatorname{Spec}(R)) \rightarrow \mathrm{Qcoh}(T)$ является вполне стро2uм.

ДокАЗАТЕЛЬство. Можно предполагать, что решето $T$ порождено семейством $\left(f_{\alpha}: \operatorname{Spec}\left(R_{\alpha}\right) \rightarrow \operatorname{Spec}(R)\right)_{\alpha \in I}$. В п. 2.7 было показано, что функтор $i^{*}$ имеет правый сопряженный функтор $i_{*}$, которьй переводит квазикогерентный модуль $\mathscr{F}$ на $T$ в квазикогерентньй пучок $\widetilde{M}$ на $\operatorname{Spec}(R)$, где модуль $M$ есть уравнитель диаграммы

$$
\prod_{\alpha \in I} \mathscr{F}\left(\operatorname{Spec}\left(R_{\alpha}\right)\right) \rightrightarrows \prod_{\alpha, \beta \in I} \mathscr{F}\left(\operatorname{Spec}\left(R_{\alpha} \otimes R_{\beta}\right)\right) \text {. }
$$

Предположим, что $i^{*}$ вполне строгий. Тогда $i_{*} i^{*}(\widetilde{M}) \cong \widetilde{M}$ для любого $R$-модуля $M$. Значит, последовательность (3.1) точна, и решето $T$ является покрывающим в канонической топологии.

Обратное утверждение сразу следует из предложения 3.3.

3.5. На категории Aff / $k$ существует очень важная субканоническая топология. Это хорошо известная плоская топология. Она порождается универсальными эффективно эпиморфными семействами $\left(f_{\alpha}: \operatorname{Spec}\left(R_{\alpha}\right) \rightarrow \operatorname{Spec}(R)\right)_{\alpha \in I}$, в которых все морфизмы $f_{\alpha}$ являются плоскими, т. е. $R_{\alpha}$ - плоские $R$-алгебры для всех $\alpha$. Плоскую топологию на категории Aff / $k$ будем обозначать ft.

3.6. Введем еще одну субканоническую топологию, которую назовем топологией эффективного спуска (edt).

Напомним вкратце теорию спуска для модулей над коммутативнњм кольцом. Пусть $f: R \rightarrow S$ - гомоморфизм коммутативных колец и $M$ - некоторый $S$-модуль. Рассмотрим два $S \otimes_{R} S$-модуля $M \otimes_{R} S$ и $S \otimes_{R} M$. Всякий $S \otimes_{R} S$-гомоморфизм $\phi: M \otimes_{R} S \rightarrow S \otimes_{R} M$ задает три $S \otimes_{R} S \otimes_{R} S$-гомоморфизма

$$
\begin{aligned}
& \phi_{1}: S \otimes_{R} M \otimes_{R} S \rightarrow S \otimes_{R} S \otimes_{R} M, \\
& \phi_{2}: M \otimes_{R} S \otimes_{R} S \rightarrow S \otimes_{R} S \otimes_{R} M, \\
& \phi_{3}: M \otimes_{R} S \otimes_{R} S \rightarrow S \otimes_{R} M \otimes_{R} S
\end{aligned}
$$

путем тензорного умножения $\phi \mathrm{c} 1_{S}$ по первой, второй и третьей компоненте соответственно. Кроме того, композиция умножения $\mu: S \otimes_{R} M \rightarrow M$ и отображения $\phi$ индуцирует гомоморфизм $S$-модулей

$$
\bar{\phi}: M \rightarrow M \otimes_{R} S \stackrel{\phi}{\rightarrow} S \otimes_{R} M \stackrel{\mu}{\rightarrow} M
$$

Под данными спуска на $S$-модуль $M$ будем понимать $S \otimes_{R} S$-гомоморфизм $\phi: M \otimes_{R} S \rightarrow S \otimes_{R} M$, для которого $\phi_{2}=\phi_{1} \phi_{3}$ и $\bar{\phi}$ является тождественньм на $M$.

Если $(M, \phi)$ и $\left(M^{\prime}, \phi^{\prime}\right)$ - две такие пары, то морфизм $g:(M, \phi) \rightarrow\left(M^{\prime}, \phi^{\prime}\right)$ между ними - это $S$-гомоморфизм $g: M \rightarrow M^{\prime}$, для которого $\left(1_{S} \otimes g\right) \phi=\phi^{\prime}\left(g \otimes 1_{S}\right)$.

Таким образом, для любого гомоморфизма коммутативных колец $f: R \rightarrow S$ получается категория данных спуска. Обозначим ее через $\operatorname{Dd}(f)$. Существует функтор $d_{f}: \operatorname{Mod}(R) \rightarrow \operatorname{Dd}(f)$, которьй переводит $R$-модуль $N$ в пару $\left(N \otimes_{R}\right.$ 
$\left.S, \phi_{N}\right)$, где $\phi_{N}$ определяется по правилу $\phi_{N}\left(n \otimes s_{1} \otimes s_{2}\right)=s_{1} \otimes n \otimes s_{2}(R$-морфизм $h: N \rightarrow N^{\prime}$ переходит в $\left.h \otimes 1_{S}\right)$.

Гомоморфизм коммутативных колец $f: R \rightarrow S$ называется гомоморфизмом әффективного спуска, если функтор $d_{f}: \operatorname{Mod}(R) \rightarrow \operatorname{Dd}(f)$ является эквивалентностью.

Обозначим через $\left(T_{f} \stackrel{i}{\hookrightarrow} \operatorname{Spec}(R)\right) \in \mathscr{C}^{-}$решето на $\operatorname{Spec}(R)$, состоящее из всех отображений $\operatorname{Spec}(B) \rightarrow \operatorname{Spec}(R)$, которые пропускаются через $f^{\text {op }}: \operatorname{Spec}(S) \rightarrow$ $\operatorname{Spec}(R)$. Как и любое решето, можно рассматривать $T_{f}$ в качестве подпредпучка множеств в $\operatorname{Spec}(R)$. Нетрудно видеть, что категория $\operatorname{Dd}(f)$ есть не что иное, как категория $\mathrm{Qcoh}\left(T_{f}\right)$ квазикогерентных модулей на $T_{f}$ и функтор $d_{f}$ совпадает с функтором обратного образа $i^{*}$.

Семейство $F=\left(f_{\alpha}: \operatorname{Spec}\left(R_{\alpha}\right) \rightarrow \operatorname{Spec}(R)\right)_{\alpha \in I}$ является семейством әффективного спуска, если функтор $i^{*}: \operatorname{Qcoh}(\operatorname{Spec}(R)) \rightarrow \mathrm{Qcoh}\left(T_{F}\right)$ есть эквивалентность, где $T_{F} \stackrel{i}{\hookrightarrow} \operatorname{Spec}(R) \in \mathscr{C}^{-}$- решето, порожденное семейством $F$.

ПРЕДЛОЖЕнИЕ 3.5. Для семейств эффективного спуска выполнены условия 1)-3) определения 1.3, т. е. они задают предтопологию на $\mathscr{C}=\mathrm{Aff} / k$.

ДокАЗАТЕЛЬСтво. В силу следствия 3.4 каждое семейство эффективного спуска является универсальньм эффективно эпиморфным семейством. Пусть $F=\left(\operatorname{Spec}\left(R_{\alpha}\right) \rightarrow \operatorname{Spec}(R)\right)_{\alpha \in I}-$ некоторое семейство эффективного спуска, а $\operatorname{Spec}(S) \rightarrow \operatorname{Spec}(R)$ - некоторый морфизм. Рассмотрим квазикогерентный модуль $\overline{\mathscr{F}}$ на решете $T_{\bar{F}}$, порожденном семейством $\bar{F}=\left(\operatorname{Spec}\left(R_{\alpha} \otimes_{R} S\right) \rightarrow\right.$ $\operatorname{Spec}(S))_{\alpha \in I}$. Определим квазикогерентный модуль $\mathscr{F}$ на $T_{F}$ по правилу

$$
\mathscr{F}(\cdot):=\overline{\mathscr{F}}\left(\cdot \times_{\operatorname{Spec}(R)} \operatorname{Spec}(S)\right)
$$

(он является прямым образом $\overline{\mathscr{F}}$ относительно канонического отображения из $T_{\bar{F}}$ в $\left.T_{F}\right)$. Поскольку $F$ является семейством эффективного спуска, найдется $R$-модуль $M$, для которого $\mathscr{F}=i_{F}^{*} \widetilde{M}$, и модуль $M$ является уравнителем пары

$$
\prod_{\alpha \in I} \overline{\mathscr{F}}\left(\operatorname{Spec}\left(S \otimes_{R} R_{\alpha}\right)\right) \rightrightarrows \prod_{\alpha, \beta \in I} \overline{\mathscr{F}}\left(\operatorname{Spec}\left(S \otimes_{R} R_{\alpha} \otimes_{R} R_{\beta}\right)\right)
$$

Следовательно, $R$-модуль $M$ имеет структуру $S$-модуля и $\overline{\mathscr{F}}=i \frac{*}{\bar{F}} \widetilde{M}$. Значит, $\bar{F}-$ семейство эффективного спуска.

Пусть $F=\left(\operatorname{Spec}\left(R_{\alpha}\right) \rightarrow \operatorname{Spec}(R)\right)_{\alpha \in I}-$ семейство эффективного спуска. Кроме того, зафиксируем семейства эффективного спуска $G_{\alpha}=\left(\operatorname{Spec}\left(R_{\beta_{\alpha}}\right) \rightarrow\right.$ $\left.\operatorname{Spec}\left(R_{\alpha}\right)\right)_{\beta_{\alpha} \in J_{\alpha}}$ для каждого $\alpha \in I$. Пусть $G$ - некоторое семейство $\left(\operatorname{Spec}\left(R_{\gamma}\right) \rightarrow\right.$

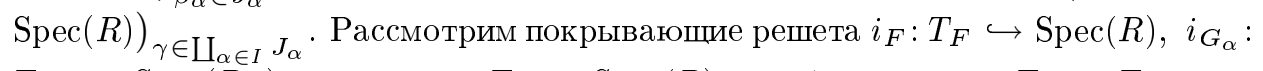
$T_{G_{\alpha}} \hookrightarrow \operatorname{Spec}\left(R_{\alpha}\right)$ и решето $i_{G}: T_{G} \hookrightarrow \operatorname{Spec}(R)$ с отображением $p: T_{G} \hookrightarrow T_{F}$.

Обозначим через $f_{\alpha}: \operatorname{Spec}\left(R_{\alpha}\right) \rightarrow T_{F}$ и $g_{\alpha}: T_{G_{\alpha}} \rightarrow T_{G}$ канонические морфизмы в категории $\mathscr{C}^{-}$. Рассмотрим диаграмму функторов

$$
\begin{array}{cc}
\operatorname{Mod}\left(T_{G_{\alpha}}, \text { can }\right) & \stackrel{j_{g \alpha}^{*}}{\longleftarrow} \operatorname{Mod}\left(T_{G}, \text { can }\right) \\
2 \uparrow_{i_{G_{\alpha}}^{*}}^{*} & \imath j_{p}^{*} \\
\operatorname{Mod}\left(\operatorname{Spec}\left(R_{\alpha}\right), \text { can }\right) & \stackrel{j_{f_{\alpha}}^{*}}{\longleftarrow} \operatorname{Mod}\left(T_{F}, \text { can }\right)
\end{array}
$$


Обе вертикальные стрелки являются эквивалентностями, потому что все семейства эффективного спуска являются покрывающими в канонической топологии.

Пусть $\mathscr{F}$ - некоторый квазикогерентный модуль на $T_{G}$. По следствию 3.2 он является пучком $\mathscr{O}_{T_{G}}$-модулей в канонической топологии. Рассмотрим пучки модулей $\mathscr{F}_{\alpha}:=j_{f_{\alpha}}^{*} j_{p *}(\mathscr{F})$. Поскольку для каждого $\alpha$ имеется изоморфизм $j_{i_{G_{\alpha}}}^{*}\left(\mathscr{F}_{\alpha}\right) \cong j_{g_{\alpha}}^{*}(\mathscr{F})$ и $G_{\alpha}$ является семейством эффективного спуска, получаем, что пучки $\mathscr{F}_{\alpha}$ является квазикогерентными. Учитывая, что решето $T_{F}$ порождено семейством $\left(f_{\alpha}\right)_{\alpha \in I}$, можно заключить, что пучок модулей $j_{p *}(\mathscr{F})$ также является квазикогерентным. Таким образом, каждый квазикогерентный модуль $\mathscr{F}$ на $T_{G}$ является подъемом квазикогерентного модуля $j_{p *}(\mathscr{F})$. Следовательно, функтор $p^{*}: \mathrm{Q} \operatorname{coh}\left(T_{F}\right) \rightarrow \mathrm{Q} \operatorname{coh}\left(T_{G}\right)$ не только вполне строгий, но и является эквивалентностью. Значит, функтор $i_{G}^{*}: \operatorname{Qcoh}(\operatorname{Spec}(R)) \rightarrow \operatorname{Qcoh}\left(T_{G}\right)$ тоже является эквивалентностью, и семейство $G$ является семейством эффективного спуска.

3.7. Топология әффективного спуска edt на категории $\mathscr{C}=$ Aff $/ k$ - это топология, для которой покрываюшими являются решета, порожденные семействами эффективного спуска. По следствию 3.4 топология edt является субканонической. Кроме того, можно показать, что топология эффективного спуска тоньше, чем плоская топология, т. е. любое покрытие в плоской топологии является покрытием и в топологии эффективного спуска. Доказано (см. [3], [6], [7]), что строго плоский гомоморфизм коммутативных колец $f: R \rightarrow S$ является морфизмом эффективного спуска. Эти доказательства распространяются и на плоские семейства.

Обозначим через $\underline{e}: \mathscr{C}^{-} \rightarrow \operatorname{Shv}(\mathscr{C}, \mathrm{edt})$ функтор ассоциирования пучка в топологии эффективного спуска.

Теорема 3.6. Пусть $X \in \mathscr{C}^{-}-$предпучок и $m: X \rightarrow \underline{e} X-$ морфизм ассоииирования пучка в топологии эффективного спуска. Тогда функтор обратного образа $m^{*}: \mathrm{Qcoh}(\underline{e} X) \rightarrow \mathrm{Q} \operatorname{coh}(X)$ является әквивалентностью.

ДокАЗАТЕЛьство. Функтор $\underline{i} \underline{e}$ изоморфен композиции $L \circ L$, где $L$ - функтор, определенный по формуле (1.2), т. е. морфизм $m$ является композицией

$$
X \stackrel{l_{X}}{\longrightarrow} L X \stackrel{l_{L X}}{\longrightarrow} \underline{e} X
$$

Таким образом, достаточно показать, что функтор $l_{X}^{*}: \mathrm{Q} \operatorname{coh}(L X) \rightarrow \mathrm{Q} \operatorname{coh}(X)$ есть эквивалентность для любого $X \in \mathscr{C}^{\wedge}$.

По следствию 3.2 категория $\mathrm{Q} \operatorname{coh}(X)$ является полной подкатегорией в категории $\operatorname{Mod}\left(X, \operatorname{can}_{X}\right)$. Функтор $l_{X}^{*}$ индуцирован функтором $j_{l_{X}}^{*}: \operatorname{Mod}\left(L X, \operatorname{can}_{L X}\right) \rightarrow$ $\operatorname{Mod}\left(X, \operatorname{can}_{X}\right)$, который, в свою очередь, является эквивалентностью. Следовательно, чтобы доказать теорему, необходимо показать, что для всякого квази-

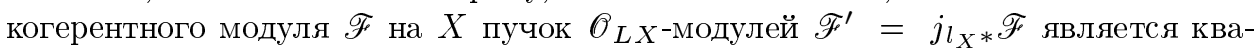
зикогерентным. Учитывая замечание 2.4 , проверим, что для всякого морфизма $s: \operatorname{Spec}(R) \rightarrow L X$ пучок $j_{s}^{*} \mathscr{F}^{\prime}$ является квазикогерентным. По определению функтора $L$ имеем

$$
L X(R)=\underset{T \in J(R)}{\underset{T \lim _{(}}{\longrightarrow}} \operatorname{Hom}_{\mathscr{C}}-(T, X)
$$


Значит, существует покрьвающее решето $i: T \hookrightarrow \operatorname{Spec}(R)$, для которого диаграмма

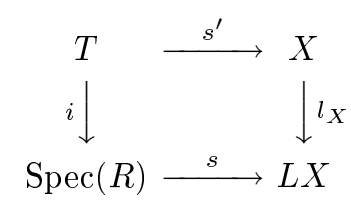

является коммутативной. Поскольку функторы $j_{l_{X}}^{*}$ и $j_{l_{X} *}$ квазиобратны друг другу по предложению 2.1 , существует изоморфизм $j_{i}^{*} j_{s}^{*} \mathscr{F}^{\prime} \cong j_{s^{\prime}}^{*} \mathscr{F}$ квазикогерентных модулей на $T$. Так как $T$ является покрываюшим решетом, функтор $j_{i}^{*}$ индуцирует эквивалентность $i^{*}: \mathrm{Q} \operatorname{coh}(\operatorname{Spec}(R)) \stackrel{\sim}{\rightarrow} \mathrm{Q} \operatorname{coh}(T)$. Следовательно, пучок $j_{s}^{*} \mathscr{F}^{\prime}$ является квазикогерентным на $\operatorname{Spec}(R)$ для всех $s: \operatorname{Spec}(R) \rightarrow L X$. Значит, $\mathscr{F}^{\prime}=j_{l_{X} *} \mathscr{F}$ - квазикогерентный модуль на $L X$.

\section{§4. Пучки в некоммутативной геометрии}

4.1. Зафиксируем базисное кольцо $k$. Обозначим через $\mathrm{Alg} / k$ категорию алгебр над базисным кольцом $k$. Если $f_{i}: R \rightarrow S_{i}, i=1,2,-$ два гомоморфизма алгебр, то копроизведение $S_{1} \coprod_{R} S_{2}$ всегда сушествует и является свободньм произведением $R$-алгебр, которое будем обозначать $S_{1} \star_{R} S_{2}$.

Через NAff / $k$ обозначим категорию противоположную к Alg $/ k$. Будем называть ее категорией некоммутативных аффинных схем. Объект NAff $/ k$, соответствующий алгебре $R$, будем обозначать через $\operatorname{Sp}(R)$. Категория некоммутативных аффинных схем имеет все расслоенные произведения, и произведение $\operatorname{Sp}\left(S_{1}\right) \times_{\mathrm{Sp}(R)} \operatorname{Sp}\left(S_{2}\right)$ есть $\operatorname{Sp}\left(S_{1} \star_{R} S_{2}\right)$.

4.2. Пусть $N:$ Aff $/ k \rightarrow$ NAff $/ k$ - естественный функтор включения, который переводит $\operatorname{Spec}(R)$ в $\operatorname{Sp}(R)$. Функтор $N$ является вполне строгим и имеет правый сопряженный функтор Com: NAff $/ k \rightarrow$ Aff $/ k$. Функтор Com переводит объект $\operatorname{Sp}(R)$ в $\operatorname{Spec}\left(R_{c}\right)$. Здесь $R_{c}$ - максимальная коммутативная факторалгебра алгебры $R$, т. е. $R_{c}=R / I$, где $I$ - идеал, порожденный всеми соотношениями вида $\langle a b-b a\rangle$.

Сушествует функтор $\widehat{N}_{!}:(\operatorname{Aff} / k)^{\wedge} \rightarrow(\text { NAff } / k)^{\wedge}$, который является продолжением $N$ на категорию предпучков (см. п. 1.2). Из п. 1.2 следует описание функтора $\widehat{N}_{\text {! в виде }}$

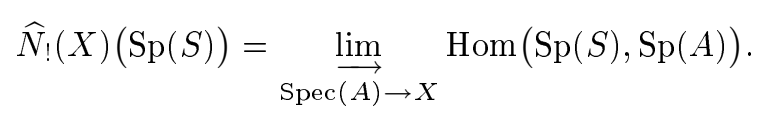

Функтор $\widehat{N}_{\text {! }}$ является вполне строгим (см. п. 1.2) и имеет правый сопряженный функтор $\widehat{N}^{*}$, ограничение которого на NAff / $k$ совпадает с функтором Com.

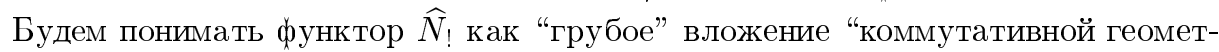
рии" в "некоммутативнуюгеометрию". Чтобы получить "тонкое" вложение, необходимо рассмотреть предпучки группоидов.

4.3. Для каждого объекта $\operatorname{Sp}(S) \in \mathrm{NAff} / k$ определим категорию $I_{S}$. Объекты $I_{S}$ - это пары $(\operatorname{Spec}(A), m)$, где $\operatorname{Spec}(A) \in \operatorname{Aff} / k$ и $m: \operatorname{Sp}(S) \rightarrow \operatorname{Sp}(A)$. Mopфизмы из $(\operatorname{Spec}(A), m)$ в $\left(\operatorname{Spec}\left(A^{\prime}\right), m^{\prime}\right)$ - это морфизмы $\xi: \operatorname{Spec}(A) \rightarrow \operatorname{Spec}\left(A^{\prime}\right)$, для которых $m^{\prime}=\xi m$. 
Каждьй морфизм $f: \operatorname{Sp}\left(S^{\prime}\right) \rightarrow \operatorname{Sp}(S)$ определяет функтор $I_{f}: I_{S} \rightarrow I_{S^{\prime}}$. Кроме того, существует функтор $\operatorname{pr}_{S}: I_{S} \rightarrow \operatorname{Aff} / k$, который переводит $(\operatorname{Spec}(A), m)$

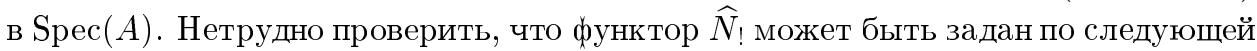
формуле:

$$
\widehat{N}_{!}(X)(\operatorname{Sp}(S))=\underset{I_{S}}{\lim } X\left(\operatorname{pr}_{S}(\cdot)\right) .
$$

4.4. Рассмотрим категорию $I_{S} / X$ объектов над $X$. Ее объекты - это тройки вида $(\operatorname{Spec}(A), m, a)$, где $m: \operatorname{Sp}(S) \rightarrow \operatorname{Sp}(A)$ и $a: \operatorname{Spec}(A) \rightarrow X$. Ее морфизмы - это такие морфизмы в $I_{S}$, которые совместимы с отображениями в $X$. Обозначим через $\operatorname{Gr}\left(I_{S} / X\right)$ группоид, ассоциированный с категорией $I_{S}$ (т. е. мы обрашаем все морфизмы). Всякий морфизм $g: \operatorname{Sp}\left(S^{\prime}\right) \rightarrow \operatorname{Sp}(S)$ задает функтор $\operatorname{Gr}\left(I_{S} / X\right) \rightarrow \operatorname{Gr}\left(I_{S^{\prime}} / X\right)$. С другой стороны, каждое отображение $f: X^{\prime} \rightarrow X$ из (Aff $/ k)^{\wedge}$ индуцирует функтор $\operatorname{Gr}\left(I_{S} / X^{\prime}\right) \rightarrow \operatorname{Gr}\left(I_{S} / X\right)$.

Теперь для объекта $X \in(\mathrm{Aff} / k)^{\wedge}$ определим предпучок группоидов $\bar{N} X \in$ $(\mathrm{NAff} / k)_{\mathrm{gr}}$ по правилу

$$
\bar{N} X(\operatorname{Sp}(S)):=\operatorname{Gr}\left(I_{S} / X\right) .
$$

Это соответствие задает функтор $\bar{N}$ из (Aff $/ k)^{\wedge}$ в категорию предпучков группоидов (NAff $/ k)_{\text {gr }}$. Из формулы (4.1) следует, что $\pi_{0}(\bar{N} X)$ изоморфен $\widetilde{N}_{!} X$. Следовательно, имеется коммутативная диаграмма функторов

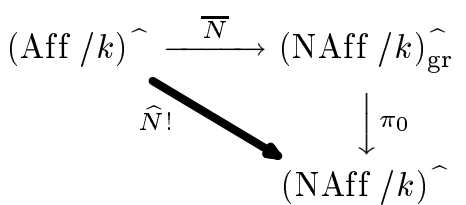

ПрЕДЛОЖенИЕ 4.1. Функтор $\bar{N}:(\text { Aff } / k)^{\wedge} \rightarrow(\text { NAff } / k)_{\mathrm{gr}}^{\wedge}$ обладает следyющими свойствами:

1) предпучок группоидов $\bar{N}(\operatorname{Spec}(A))$ изоморфен пучку множеств $N(\operatorname{Spec}(A))=\operatorname{Sp}(A)$, m. е. ограничение функтора $\bar{N}$ на категорию Aff $/ k$ изоморфно $N$;

2) для каждого обгекта $\operatorname{Spec}(A) \in \operatorname{Aff} / k$ группоид $\bar{N} X(\operatorname{Sp}(A))$ эквивалентен множеству $X(\operatorname{Spec}(A))$;

3) функтор $\bar{N}$ является вполне строгим.

ДокАЗАТЕЛЬСТво. Группоид, ассоциированный с категорией, в которой имеется финальньй или начальный объект, эквивалентен одноточечному множеству. Отсюда получаем, что $\operatorname{Gr}\left(I_{S} / \operatorname{Spec}(A)\right)$ эквивалентен $\operatorname{Hom}_{\mathrm{NAff}}(\operatorname{Sp}(S), \operatorname{Sp}(A))$ для всякой алгебры $S$ и коммутативной алгебры $A$. Следовательно, $\bar{N}(\operatorname{Spec}(A)) \cong$ $\operatorname{Sp}(A)$ и свойство 1) выполнено. По тем же причинам группоид $\operatorname{Gr}\left(I_{A} / X\right)$ эквивалентен множеству $X(\operatorname{Spec}(A))$ для каждой коммутативной алгебры $A$. Это доказывает свойство 2).

Поскольку функтор $\widehat{N}_{!}=\pi_{0} \bar{N}$ вполне строгий (см. п. 4.2), функтор $\bar{N}$ является строгим. Известно, что композиция $\widehat{N}^{*} \pi_{0} \bar{N}$ изоморфна тождественному функтору на (Aff $/ k)^{\wedge}$.

Чтобы доказать полноту функтора $\bar{N}$, необходимо проверить, что любой морфизм $f: \bar{N} Y \rightarrow \bar{N} X$ лежит в его образе. Морфизм $f$ определяется заданием морфизмов $f_{S}: \operatorname{Gr}\left(I_{S} / Y\right) \rightarrow \operatorname{Gr}\left(I_{S} / X\right)$ для всех $S \in \operatorname{Alg} / k$, которые совместимы 
друг с другом. Пусть $t=(\operatorname{Spec}(A), m, a)$ - некоторьй элемент $\operatorname{Gr}\left(I_{S} / Y\right)$, где $m: \operatorname{Sp}(S) \rightarrow \operatorname{Sp}(A)$ и $a \in Y(\operatorname{Spec}(A))$. Морфизм $f_{S}$ переводит этот элемент в $t^{\prime}=\left(\operatorname{Spec}(A), m, a^{\prime}\right) \in \operatorname{Gr}\left(I_{S} / X\right)$, где $a^{\prime} \in X(\operatorname{Spec}(A))$. Поскольку $f_{S}$ и $f_{A}$ совместимы, получаем, что $a^{\prime}=f_{A}(a)$. Следовательно, зная морфизм $f_{A}$, можно найти элемент $a^{\prime}$. Таким образом, каждый морфизм $f_{S}$ однозначно определяется множеством морфизмов $f_{A}$ с коммутативньми алгебрами $A$. Отсюда получаем, что морфизм $f$ совпадает с морфизмом $\bar{N} \widehat{N}^{*} \pi_{0}(f)$ и, значит, лежит в образе $\bar{N}$, т.е. функтор $\bar{N}$ является вполне строгим.

4.5. Пусть $X$ - некоторый объект из $(\mathrm{Aff} / k)^{\wedge}$, с которымможно связать две категории Aff / $X$ и NAff $/ \bar{N} X$ (определение второй было дано в п. 2.10). Существует функтор из Aff $/ X$ в NAff $/ \bar{N} X$, который переводит элемент $a \in X(\operatorname{Spec}(A))$ в элемент $(\operatorname{Spec}(A), \mathrm{id}, a) \in \operatorname{Gr}\left(I_{\operatorname{Spec}(A)} / X\right)$ (см. п. 4.4). Обозначим этот функтор через $\bar{n}_{X}$. Он индуцирует функтор обратного образа $\bar{n}_{X}^{*}: \operatorname{Qcoh}_{r}(\bar{N} X) \rightarrow \mathrm{Qcoh}(X)$.

Сопоставление предпучка множеств $X \in(\mathrm{Aff} / k) \wedge$ с предпучком группоидов $\bar{N} X \in(\mathrm{NAff} / k)_{\text {gr }}$ рассматривается как “тонкое" вложение коммутативной геометрии в некоммутативную в силу того, что выполнено следующее свойство.

Tеорема 4.2. Пусть $X-$ обгект из $(\operatorname{Aff} / k)^{\wedge}$. Функтор $\bar{n}_{X}^{*}: \operatorname{Qcoh}_{r}(\bar{N} X) \rightarrow$ $\mathrm{Q} \operatorname{coh}(X)$, индуцированный естественньмм функтором $\bar{n}_{X}:$ Aff $/ X \rightarrow$ NAff / $\bar{N} X$, является әквивалентностью.

ДокАЗАТЕЛЬСтво. Построим функтор, квазиобратньй к функтору $\bar{n}_{X}^{*}$. Для каждого объекта $X \in \operatorname{Aff} / k$ определим предпучок категорий $C X$ на NAff $/ k$ (т. е. контравариантный функтор из NAff / $k$ в категорию всех малых категорий) по правилу

$$
C X(\operatorname{Sp}(S)):=I_{S} / X
$$

Рассмотрим категорию NAff $/ C X$, определенную в п. 2.10. Кроме канонического функтора из Aff / $X$ в NAff / $C X$, сушествует функтор $\kappa_{X}$ из NAff $/ C X$ в Aff $/ X$, который переводит элемент $t=(\operatorname{Spec}(A), m, a) \in I_{S} / X$ в элемент $a \in X(\operatorname{Spec}(A))$, где $m: \operatorname{Sp}(S) \rightarrow \operatorname{Spec}(A)$.

Пусть $\mathscr{F}$ - квазикогерентный модуль на $X$. Можно определить предпучок $\mathscr{G}$ на категории NAff / $C X$ по правилу

$$
\mathscr{G}(t)=\mathscr{F}(a) \otimes_{(A, m)} S .
$$

Более формально, $\kappa_{X}$ индуцирует функтор $\widehat{\kappa}_{X}^{*}:(\operatorname{Aff} / X)^{\wedge} \rightarrow(\mathrm{NAff} / C X)^{\wedge}(\mathrm{cm}$. п. 1.2). Тогда предпучок $\mathscr{G}$ можно задать как $\widehat{\kappa}_{X}^{*}(\mathscr{F}) \otimes_{\widehat{\kappa}_{X}^{*}\left(\mathscr{O}_{X}\right)} \mathscr{O}_{C X}$.

Далее, морфизм $\alpha: t \rightarrow t^{\prime}$ в категории $I_{S} / X$ есть по определению морфизм $\alpha: \operatorname{Spec}(A) \rightarrow \operatorname{Spec}\left(A^{\prime}\right)$, для которого $\alpha m=m^{\prime}$ и $a^{\prime} \alpha=a$. Всякий такой морфизм задает изоморфизм между $\mathscr{G}(t)$ и $\mathscr{G}\left(t^{\prime}\right)$. Действительно,

$$
\mathscr{G}(t)=\mathscr{F}(a) \otimes_{(A, m)} S \cong \mathscr{F}\left(a^{\prime}\right) \otimes_{\left(A^{\prime}, \alpha\right)} A \otimes_{(A, m)} S \cong \mathscr{F}\left(a^{\prime}\right) \otimes_{\left(A^{\prime}, m^{\prime}\right)} S=\mathscr{G}\left(t^{\prime}\right) .
$$

Следовательно, предпучок $\mathscr{G}$ индуцирован некоторьм предпучком $\mathscr{H}$ на $\bar{N} X$ относительно канонического морфизма NAff $/ C X \rightarrow \mathrm{NAff} / \bar{N} X$. Кроме того, очевидно, что $\mathscr{H}$ является квазикогерентным модулем. Аналогично определяется отображение на морфизмах между квазикогерентными модулями. Окончательно получаем функтор $\bar{n}_{* X}$ из $\mathrm{Q} \operatorname{coh}(X)$ в $\mathrm{Qcoh}_{r}(\bar{N} X)$, который является квазиобратньм к $\bar{n}_{X}^{*}$. 
4.6. Далее будем записывать $\mathscr{C}$ вместо NAff $/ k$. Обозначим через $\Phi=(\mathscr{C}$, can $)$ сайт, окольцованный пучком $\mathscr{O}$. Пусть $X$ - некоторый объект из $\mathscr{C}$, и $j_{X}: \mathscr{C} / X \rightarrow$ $\mathscr{C}$ - канонический функтор. Как и ранее, обозначим через $\operatorname{can}_{X}$ каноническую топологию на $\mathscr{C} / X$. Она индуцирована канонической топологией на $\mathscr{C}$ (см. п. 1.5). Рассмотрим окольцованный сайт $\Phi_{X}=\left(\mathscr{C} / X, \operatorname{can}_{X}\right)$ с пучком колец $\mathscr{O}_{X}$. В коммутативной ситуации мы знаем, что всякий квазикогерентный модуль на $X$ является пучком в канонической топологии на $\mathscr{C} / X$ (см. п. 3.2). Аналогичное утверждение в некоммутативной ситуации также верно, но доказательство не является таким прямым, как в коммутативной.

Tеорема 4.3. Пусть $X-$ предпучок множсеств на категории $\mathscr{C}=\mathrm{NAff} / k$. Тогда всякий квазикогерентный модуль $\mathscr{M}$ на $X$ является пучком в канонической топологии на NAff / $X$.

ДоказАТЕЛЬство. Пусть $A-k$-алгебра и $\left(f_{\alpha}: A \rightarrow B_{\alpha}\right), \alpha \in I,-$ семейство морфизмов алгебр такое, что соответствуюшее семейство отображений $f_{\alpha}^{\text {op }}$ в противоположной категории NAff / $k$ является покрывающим в канонической топологии. Для доказательства теоремы необходимо показать, что для любого (правого) $A$-модуля $M$ гомоморфизм $\varepsilon_{M}$ в диаграмме

$$
M \stackrel{\varepsilon_{M}}{\longrightarrow} \prod_{\alpha \in I} M \otimes_{A} B_{\alpha} \rightrightarrows \prod_{\alpha, \beta \in I} M \otimes_{A}\left(B_{\alpha} \star_{A} B_{\beta}\right)
$$

является уравнителем.

Обозначим через $N A$-бимодуль $A \otimes_{\mathbb{Z}} M$. Определим кольцо $S$ как пространство $A \oplus N$ с умножением $\left(a_{1}, n_{1}\right)\left(a_{2}, n_{2}\right)=\left(a_{1} a_{2}, a_{1} n_{2}+n_{1} a_{2}\right)$. По лемме 4.4 каноническое отображение $\phi: M \rightarrow N$ является вложением. Обозначим через $i$ вложение $N$ в $S$ и через $\psi_{\alpha}-$ канонические отображения $M \otimes_{A} B_{\alpha} \rightarrow S \star_{A} B_{\alpha}$ для каждого $\alpha \in I$. Пусть $\psi$ - произведение $\psi_{\alpha}$ по всем $\alpha \in I$.

Рассмотрим коммутативную диаграмму

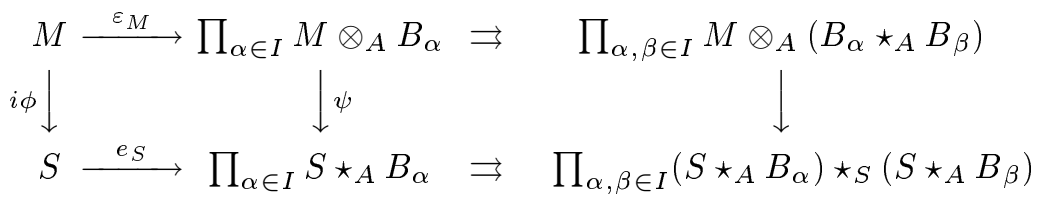

Поскольку семейство $\left(f_{\alpha}^{\text {op }}\right)$ является покрывающим, отображение $e_{S}$ является уравнителем. Кроме того, отображения $\phi$ и $e_{S}-$ вложения, следовательно, отображение $\varepsilon_{M}$ также является вложением. Получаем, что каждый квазикогерентный модуль есть отделимьй предпучок.

Предположим, что выполнены следующие условия:

1) отображения $\psi_{\alpha}-$ вложения для любого $\alpha$;

$2)$ пересечение $e_{S}(S)$ и $\psi\left(\prod_{\alpha} M \otimes_{A} B_{\alpha}\right)$ совпадает с $e_{S} i \phi(M)$.

Тогда получаем, что $\varepsilon_{M}$ является уравнителем.

Таким образом, чтобы закончить доказательство, необходимо проверить, что условия 1) и 2) выполнены.

1) Обозначим $A$-бимодуль $B_{\alpha} / A$ через $\bar{B}_{\alpha}$. Рассмотрим градуированное кольцо $R_{\alpha}$ для всякого $\alpha \in I$, определенное по правилу

$$
R_{\alpha}=B_{\alpha} \oplus\left(B_{\alpha} \otimes_{A} N \otimes_{A} B_{\alpha}\right) \oplus\left(B_{\alpha} \otimes_{A} N \otimes_{A} \bar{B}_{\alpha} \otimes_{A} N \otimes_{A} B_{\alpha}\right) \oplus \cdots
$$


с законом умножения

$$
\begin{aligned}
&\left(b_{0} \otimes n_{1} \otimes\right.\left.\bar{b}_{1} \otimes \cdots \otimes \bar{b}_{i-1} \otimes n_{i} \otimes b_{i}\right) \\
& \times\left(b_{i}^{\prime} \otimes n_{i+1} \otimes \bar{b}_{i+1} \otimes \cdots \otimes \bar{b}_{i+j-1} \otimes n_{i+j} \otimes b_{i+j}\right) \\
&=\left(b_{0} \otimes n_{1} \otimes \bar{b}_{1} \otimes \cdots \otimes \bar{b}_{i-1} \otimes n_{i} \otimes \bar{b}_{i} b_{i}^{\prime} \otimes n_{i+1}\right. \\
&\left.\otimes \bar{b}_{i+1} \otimes \cdots \otimes \bar{b}_{i+j-1} \otimes n_{i+j} \otimes b_{i+j}\right)
\end{aligned}
$$

Существуют гомоморфизмы колец $B_{\alpha} \rightarrow R_{\alpha}$ и $S \rightarrow R_{\alpha}$. Следовательно, сушествует канонический гомоморфизм $S \star_{A} B_{\alpha} \rightarrow R_{\alpha}$. По лемме 4.4 отображение

$$
M \otimes_{A} B_{\alpha} \rightarrow B_{\alpha} \otimes_{A} N \otimes_{A} B_{\alpha} \cong B_{\alpha} \otimes_{\mathbb{Z}} M \otimes_{A} B_{\alpha}
$$

является вложением. Значит, отображение $M \otimes_{A} B_{\alpha} \rightarrow R_{\alpha}$ также есть вложение. Оно является композицией $\psi_{\alpha}$ с каноническим отображением $S \star_{A} B_{\alpha} \rightarrow R_{\alpha}$. Таким образом, получаем, что $\psi_{\alpha}-$ вложение для всякого $\alpha \in I$.

2) Отображение $i$ является вложением $N$ в $S$. Чтобы проверить второе условие, достаточно показать, что пересечение $e_{S} i(N)$ и $\psi\left(\prod_{\alpha \in I} M \otimes_{A} B_{\alpha}\right)$ совпадает с $e_{S} i \phi(M)$. Заметим, что отображения $e_{S} i$ и $\psi$ пропускаются через каноническое отображение

$$
\prod_{\alpha \in I} B_{\alpha} \otimes_{A} N \otimes_{A} B_{\alpha} \rightarrow \prod_{\alpha \in I} S \star_{A} B_{\alpha}
$$

С другой стороны, существует сквозное отображение

$$
\prod_{\alpha \in I} S \star_{A} B_{\alpha} \rightarrow \prod_{\alpha \in I} R_{\alpha} \rightarrow \prod_{\alpha \in I} B_{\alpha} \otimes_{A} N \otimes_{A} B_{\alpha}
$$

которое расщепляет предыдущее. Следовательно, $\prod_{\alpha \in I} B_{\alpha} \otimes_{A} N \otimes_{A} B_{\alpha}$ является прямым слагаемым объекта $\prod_{\alpha \in I} S \star_{A} B_{\alpha}$.

Обозначим через $\rho$ и $\theta$ отображения $N \rightarrow \prod_{\alpha \in I} B_{\alpha} \otimes_{A} N \otimes_{A} B_{\alpha}$ и $\prod_{\alpha \in I} M \otimes_{A}$ $B_{\alpha} \rightarrow \prod_{\alpha \in I} B_{\alpha} \otimes_{A} N \otimes_{A} B_{\alpha}$ соответственно. Существует коммутативная диаграмма

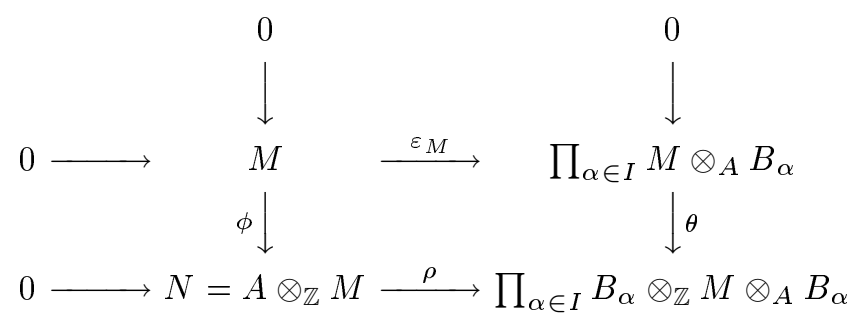

По лемме 4.4 вложения $\phi$ и $\theta$ расщепляются как гомоморфизмы абелевых групп. Более того, легко видеть, что эти расщепления коммутируют. Следовательно, пересечение $\rho(N)$ и $\theta\left(\prod_{\alpha \in I} M \otimes_{A} B_{\alpha}\right)$ совпадает с $\rho \phi(M)$. Это доказывает условие 2) и, значит, теорему.

Лемма 4.4. Пусть $R$ - кольцо и $M-$ правый $R$-модуль. Тогда каноническое отображсение $M \rightarrow R \otimes_{\mathbb{Z}} M$ является вложсением правых $R$-модулей $и$ расщепляется как морфизм абелевых групп. 
ДокАЗАТЕЛЬСтво. Очевидно, что композиция отображений абелевых групп

$$
M \rightarrow R \otimes_{\mathbb{Z}} M \stackrel{\sim}{\rightarrow} M \otimes_{\mathbb{Z}} R \rightarrow M
$$

является тождественньм отображением. (Здесь последнее отображение есть действие кольца $R$ на правый модуль $M$.) Следовательно, $M \rightarrow R \otimes_{\mathbb{Z}} M$ является вложением абелевых групп и, значит, вложением правых $R$-модулей.

СЛЕДСТВИЕ 4.5. Пусть $X-$ предпучок множеств на категории $\mathscr{C}=$ NAff $/ k$. Тогда категория квазикогерентных модулей $\mathrm{Qcoh}_{r}(X)$ является полной подкатегорией в категории пучков в канонической топологии $\operatorname{Mod}_{r}\left(X, \operatorname{can}_{X}\right) . \quad$ Eсли $m: X \rightarrow \underline{c} X-$ морфизм ассоциирования пучка в канонической топологии, то функтор обратного образа $m^{*}: \operatorname{Qcoh}_{r}(\underline{c} X) \rightarrow$ $\mathrm{Qcoh}_{r}(X)$ является вполне строгим.

Это утверждение следует из теоремы 4.3 и предложения 2.1.

\section{Список литературы}

1. Artin M., Grothendieck A., Verdier J. L. Théorie des Topos et Cohomologie Etale des Schémas // SGA4. Lecture Notes in Math. V. 269. Heidelberg: Springer, 1972.

2. Berthelot P., Grothendieck A., Illusie L. Théorie des intersections et théoreme de Riemann-Roch // SGA6. Lect. Notes in Math. V. 225. Heidelberg: Springer, 1971.

3. Grothendieck A. Revetements étale et groups fondamental // SGA1. Lecture Notes in Math. V. 224. Heidelberg: Springer, 1971.

4. Faith C. Algebra: Rings, Modules and Categories. I. Berlin-Heidelberg-N.Y.: Springer, 1973.

5. Mac Lane S., Moerdijk I. Sheaves in Geometry and Logic. Berlin-Heidelberg-N.Y.: Springer, 1992.

6. Knus M.A., Ojanguren M. Théorie de la Descente et Algèbres d'Azumaya // Lect. Notes in Math. V. 389. Heidelberg: Springer, 1974.

7. Murre J. Lectures on an Introduction to Grothendieck's Theory of the Fundamental Group // Lect. Notes. V. 40. Bombay: Tata Institute of Fundamental Research, 1967. 